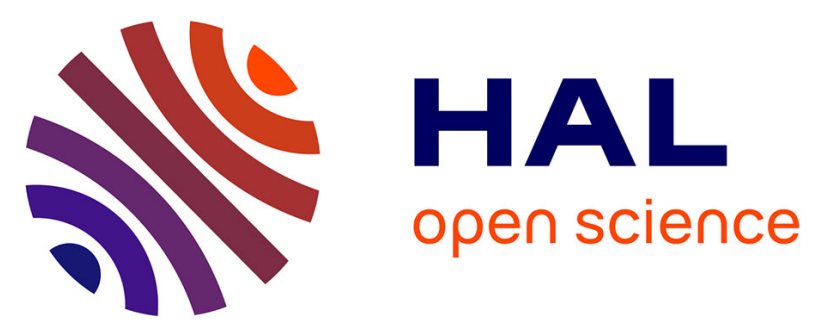

\title{
A nash game algorithm for the solution of coupled conductivity identification and data completion in cardiac electrophysiology
}

Rabeb Chamekh, Abderrahmane Habbal, Moez Kallel, Nejib Zemzemi

\section{To cite this version:}

Rabeb Chamekh, Abderrahmane Habbal, Moez Kallel, Nejib Zemzemi. A nash game algorithm for the solution of coupled conductivity identification and data completion in cardiac electrophysiology. Mathematical Modelling of Natural Phenomena, 2019, Mathematical modelling in cardiology, 14 (2), pp.15. 10.1051/mmnp/2018059 . hal-01923819v2

\section{HAL Id: hal-01923819 \\ https://hal.science/hal-01923819v2}

Submitted on 18 Feb 2019

HAL is a multi-disciplinary open access archive for the deposit and dissemination of scientific research documents, whether they are published or not. The documents may come from teaching and research institutions in France or abroad, or from public or private research centers.
L'archive ouverte pluridisciplinaire HAL, est destinée au dépôt et à la diffusion de documents scientifiques de niveau recherche, publiés ou non, émanant des établissements d'enseignement et de recherche français ou étrangers, des laboratoires publics ou privés. 


\title{
A NASH GAME ALGORITHM FOR THE SOLUTION OF COUPLED CONDUCTIVITY IDENTIFICATION AND DATA COMPLETION IN CARDIAC ELECTROPHYSIOLOGY
}

\author{
Rabeb Chamekh ${ }^{1}$, Abderrahmane Habbal $^{2, *}$, Moez Kallel $^{1}$ \\ AND NEJIB ZEMZEMI ${ }^{3,4}$
}

\begin{abstract}
We consider the identification problem of the conductivity coefficient for an elliptic operator using an incomplete over specified measures on the surface. Our purpose is to introduce an original method based on a game theory approach, and design a new algorithm for the simultaneous identification of conductivity coefficient and data completion process. We define three players with three corresponding criteria. The two first players use Dirichlet and Neumann strategies to solve the completion problem, while the third one uses the conductivity coefficient as strategy, and uses a cost which basically relies on an identifiability theorem. In our work, the numerical experiments seek the development of this algorithm for the electrocardiography imaging inverse problem, dealing with inhomogeneities in the torso domain. Furthermore, in our approach, the conductivity coefficients are known only by an approximate values. We conduct numerical experiments on a $2 \mathrm{D}$ torso case including noisy measurements. Results illustrate the ability of our computational approach to tackle the difficult problem of joint identification and data completion.
\end{abstract}

Mathematics Subject Classification. 35J25, 35N05, 91A80.

Accepted October 2, 2018.

\section{INTRODUCTION}

We consider an open bounded domain $\Omega$ in $\mathbb{R}^{d}(d=2$ or 3$)$ with a sufficiently smooth boundary $\partial \Omega$ composed of two connected disjoint components $\Gamma_{c}$ and $\Gamma_{i}$.

Given a flux $\phi$ and a potential $f$ on $\Gamma_{c}$, inverse problems are in general concerned by finding the corresponding flux and potential on the remaining part of the boundary $\Gamma_{i}$ and the conductivity on $\Omega$.

Thus, the generic formulation of the problem is set as follows.

Keywords and phrases: Nash algorithm, data completion, conductivity identification.

${ }^{1}$ Université de Tunis El Manar, Ecole Nationale d'Ingénieurs de Tunis, LAMSIN, BP 37, 1002 Tunis Belvedere, Tunisia.

${ }^{2}$ Université Côte d'Azur, INRIA, CNRS, LJAD, UMR 7351, Parc Valrose, 06108 Nice, France.

3 INRIA, Bordeaux - Sud-Ouest, 200 avenue de la vielle tour, 33405 Talence Cedex, France.

${ }^{4}$ IHU LIRYC, Pessac, France.

* Corresponding author: habbal@polytech.unice.fr 
Find $(\eta, \tau, k) \in \mathbb{H}^{-1 / 2}\left(\Gamma_{i}\right) \times \mathbb{H}^{1 / 2}\left(\Gamma_{i}\right) \times \mathbb{L}(\Omega)$ such that there exist a potential $u$ satisfying:

$$
\begin{cases}\operatorname{div}(k \nabla(u))=0 & \text { in } \Omega, \\ u=f & \text { on } \Gamma_{c} \\ k \nabla(u) \cdot n=\phi & \text { on } \Gamma_{c}\end{cases}
$$

where $\mathbb{L}(\Omega)=\left\{k \in \mathbb{L}^{\infty}(\Omega) ; \quad k \geq k_{\min }>0\right\}$. The data completion problem is an ill-posed inverse problem in the sense of Hadamard [20], whose purpose is the reconstruction of boundary conditions on the inaccessible part of the domain's boundary from overspecified data on a part of the boundary. This problem is already discussed in literature by several methods and various algorithms have been proposed like optimal control method [1], minimizing an energy-like error functional [5] and the alternating algorithm presented by Kozlov et al. [24]. In [12], the authors show how to recover the missing data from the Cauchy over specified data using iterated Tikhonov regularization. Steklov-Poincaré formulation with a Tikhonov and a Lavrentiev regularization was used in $[8,9]$ to solve the data completion problem. In articles of Habbal and Kallel [18, 19], a Nash game approach is used to solve the data completion problem. Furthermore, the authors compare the Nash algorithm to a control type method and prove its efficiency. In another paper [22], the Nash game approach is used to solve the image inpainting problem as a nonlinear Cauchy problem. The identification of conductivity parameter has also been investigated in many studies. Among them, we can mention the work of Brown [11] that gives a formula for recovering the conductivity from the Dirichlet to Neumann map and the work of Nachman et al. [25] in which they use a known current density field in the interior of the domain.

In most of the studies, these variabilities of the conductivity values have not been considered. In particular, the torso is assumed in the literature, in most of the studies to be homogenous. Moreover, when the conductivity heterogeneities are included, they are determined from data obtained from textbooks. The problem is that the difference between the experiment environments and other factors related to the measurement tools make this data to be different from a paper to another $[14,15]$.

Only few works have evaluated the effect of conductivities variabilities in the propagation of the electrical potential on the body surface $[28,29]$. A more rigorous work to evaluate the uncertainties of the forward problem solution as a consequence of the conductivity uncertainties has been done in [16], where the authors use the stochastic finite elements method (SFEM) to describe the effect of lungs muscles and fat conductivities. In [29], a principal component approach has been used to predict the effect of conductivities variation on the body surface potential. As far as the inverse problem is concerned, authors in [3] studied the effect of the conductivity uncertainties on the accuracy of the ECGI inverse solution using the stochastic optimal control approach based on SFEM to solve the inverse problem. In that work, there was no aim to estimate the conductivity values. The identification of the conductivities of the torso using a single measurement has been subject of a numerical study [10] using synthetic data, or using on in vivo data [7]. However, none of these works coupled the conductivity optimization to the data completion problem. In this work, our main contribution is to couple the two inverse problems: we aim at simultaneously identifying the missing boundary data on the inaccessible part of the boundary and the values of the conductivities in the torso domain from data measured over the accessible boundary.

One of the difficult questions is the identifiability of conductivity problem. There exist a few results that treats this problem $[17,23,26,27]$ that use either an infinity data on the border or a set of observations in the domain.

In the present paper, we introduce an original method based on a game theory approach to solve our coupled inverse problem. This method has wide applications ranging from bioelectrical field to mechanical engineering. Here, our numerical experiments target the medical applications related to the non-invasive electrocardiography imaging.

The thorax contains several tissues like lung, bones and fat. Each one is characterized by a conductivity whose value varies from an organ to another and also varies from one person to another. The aim of our work is to reconstruct the potential and the flux on the surface of the heart from measurements on the surface of 
the thorax, in addition improve the value of the conductivity of different tissues. In Section 1, we talk about the identifiability problem. In Section 2, we present our approach for the solution of the coupled problem of conductivity identification and data completion. Our algorithm is provided in Section 3 where we also present an adjoint state method to compute the gradient of our costs. Finally, in Section 4, we present two numerical experiments which illustrate the efficiency of our method and its robustness with respect to noisy data. The main conclusion of the study is finally presented in Section 5.

\section{IDENTIFIABILITY OF THE CONDUCTIVITY}

The problem of identifiability of the conductivity amounts to seek for the conditions under which one is able to guarantee the uniqueness of the actual conductivity parameter. Usually, an equivalent formulation is to say that different values of the conductivity must generate different solutions of the problem. The identifiability of the found conductivity is not always guaranteed. Therefore, this difficult problem was investigated by many authors (see e.g. $[17,26]$ ). One of the methods to prove it is to use Dirichlet-to-Neumann application defined as follows:

$$
\begin{gathered}
\Lambda_{k}: \mathbb{H}^{1 / 2}(\partial \Omega) \rightarrow \mathbb{H}^{-1 / 2}(\partial \Omega) \\
u_{\mid \partial \Omega} \mapsto(k \nabla(u) . n)_{\mid \partial \Omega}
\end{gathered}
$$

where $k \in \mathbb{L}(\Omega)$ and $u$ is a solution of a Dirichlet problem, which means the need for an infinite number of measurements on the boundary.

Here, our numerical experiments target electrocardiography applications. The domain $\Omega$ models a thorax, composed of many subdomains $\Omega=\bigcup_{j=1}^{n} \Omega_{j}$. The conductivity $k$ of the domain is assumed to be piecewise constant. The identifiability of $k$ using a unique boundary measurement is given by the following theorem.

Theorem 2.1. Let $\Omega$ be a domain such that $\Omega=\bigcup_{j=1}^{n} \Omega_{j}$. Each $\Omega_{j}$ is characterized by a constant conductivity $k_{j}$ and the value of $k_{1}$ is assumed to be known in $\Omega_{1}$, see Figure 1.

If $k^{(1)}$ and $k^{(2)}$ are two conductivities which fulfill the Cauchy problem:

$$
\begin{cases}\operatorname{div}\left(k^{(p)} \nabla\left(u^{(p)}\right)\right)=0 & \text { in } \Omega, \\ u^{(p)}=f & \text { on } \Gamma_{\mathrm{c}}, \\ k^{(p)} \nabla\left(u^{(p)}\right) \cdot n=\phi & \text { on } \Gamma_{\mathrm{c}}\end{cases}
$$

where $p=1,2$ and the potential $f$ is not a constant on $\Gamma_{c}$, then $k_{j}^{(1)}=k_{j}^{(2)}$ in $\Omega_{j} \forall j$.

Proof. In $\Omega_{1}, k^{(1)}=k^{(2)}=k_{1}$. Let us consider $u^{(p)}, p \in\{1,2\}$, the solution of the Cauchy problem in $\Omega_{1}$, it satisfies the following equation

$$
\begin{cases}\operatorname{div}\left(k_{1} \nabla\left(u^{(p)}\right)\right)=0 & \text { in } \Omega_{1}, \\ u^{(p)}=f & \text { on } \Gamma_{c}, \\ k_{1} \nabla\left(u^{(p)}\right) \cdot n=\phi & \text { on } \Gamma_{c} .\end{cases}
$$

Using the Holmgren's theorem [13] and the continuity of flux on the boundary of $\Omega_{2}$, denoted by $\Gamma_{2}$, we obtain the equality of the potentials and the flux on $\Gamma_{2}$ :

$$
\left\{\begin{array}{l}
u^{(1)}=u^{(2)}=f_{2} \\
k_{1} \nabla\left(u^{(1)}\right) \cdot n=k_{1} \nabla\left(u^{(2)}\right) \cdot n=\phi_{2} .
\end{array}\right.
$$




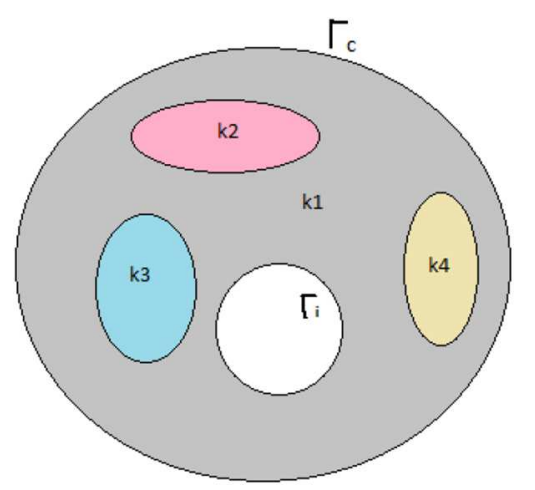

FIGURE 1. The conductivity domain.

In $\Omega_{2}, u^{(p)}$ fulfills the following direct problem:

$$
\begin{cases}\operatorname{div}\left(k_{2}^{(p)} \nabla\left(u^{(p)}\right)\right)=0 & \text { in } \Omega_{2} \\ u^{(p)}=f_{2} & \text { on } \Gamma_{2}\end{cases}
$$

where $k_{2}^{(p)}, p \in\{1,2\}$, is the constant, so we can simplify and we get $u^{(1)}=u^{(2)}$ in $\Omega_{2}$.

From the continuity of the flux and the transmission conditions on $\Gamma_{2}$, we have

$$
k_{2}^{(1)} \nabla\left(u^{(1)}\right) \cdot n=k_{2}^{(2)} \nabla\left(u^{(2)}\right) \cdot n=\phi_{2} .
$$

If $\nabla\left(u^{(p)}\right) . n \neq 0$, we conclude that $k_{2}^{(1)}=k_{2}^{(2)}$ in $\Omega_{2}$ and we prove the identifiability.

We suppose that we have $\nabla\left(u^{(p)}\right) \cdot n=0$ on $\Gamma_{2}$, so $u^{p}$ verify:

$$
\begin{cases}\operatorname{div}\left(k_{2}^{(p)} \nabla\left(u^{(p)}\right)\right)=0 & \text { in } \Omega_{2} \\ k_{2}^{(p)} \nabla\left(u^{(p)}\right) \cdot n=0 & \text { on } \Gamma_{2} .\end{cases}
$$

The constant potential is a solution of this direct problem in $\Omega_{2}$. From the continuity theorem, we conclude that the potential $u$ is constant in $\Omega$, which is absurd because $f$ is generically not constant on $\Gamma_{c}$.

\section{A game formulation of the COUPlED PROBlem of CONDUCTIVity IDENTIFICATION AND DATA COMPLETION}

We start by defining the admissible space $\mathbb{A}_{a d}$ of the conductivity functions we look for.

$$
\mathbb{A}_{a d}=\left\{k \text { piecewise real value constants, such that } k_{\min } \leq k \leq k_{\max } \text { in } \Omega \text { and } k=k_{1} \text { in } \Omega_{1}\right\},
$$

where the lower and upper bounds of the conductivity values $k_{\min }$ and $k_{\max }$, respectively, are non-negative constants.

We assume that the Cauchy data $f$ and $\phi$ belongs to $\mathbb{H}^{1 / 2}\left(\Gamma_{c}\right) \times \mathbb{H}^{-1 / 2}\left(\Gamma_{c}\right)$. For given potential $\tau \in \mathbb{H}^{1 / 2}\left(\Gamma_{i}\right)$, flux $\eta \in \mathbb{H}^{-1 / 2}\left(\Gamma_{i}\right)$ and conductivity $k \in \mathbb{A}_{a d}$ we define $u_{1}:=u_{1}(\eta, k), u_{2}:=u_{2}(\tau, k)$ and $u_{3}:=u_{3}(\tau, k)$ as the 
respective solutions to the following three boundary value sub-problems:

$$
(S P 1)\left\{\begin{array} { l l } 
{ \operatorname { d i v } ( k \nabla ( u _ { 1 } ) ) = 0 } & { \text { in } \Omega , } \\
{ u _ { 1 } = f } & { \text { on } \Gamma _ { c } , } \\
{ k \nabla ( u _ { 1 } ) \cdot n = \eta } & { \text { on } \Gamma _ { i } . }
\end{array} \quad ( S P 2 ) \left\{\begin{array} { l l } 
{ \operatorname { d i v } ( k \nabla ( u _ { 2 } ) ) = 0 } & { \text { in } \Omega , } \\
{ u _ { 2 } = \tau } & { \text { on } \Gamma _ { i } , } \\
{ k \nabla ( u _ { 2 } ) \cdot n = \phi } & { \text { on } \Gamma _ { c } . }
\end{array} \quad ( S P 3 ) \left\{\begin{array}{ll}
\operatorname{div}\left(k \nabla\left(u_{3}\right)\right)=0 & \text { in } \Omega, \\
u_{3}=\tau & \text { on } \Gamma_{i}, \\
u_{3}=f & \text { on } \Gamma_{c} .
\end{array}\right.\right.\right.
$$

To solve our inverse problem, we use the game theory, which studies the strategic interactions between players.

Let us present the following three costs: for any $\eta \in \mathbb{H}^{-1 / 2}\left(\Gamma_{i}\right), \tau \in \mathbb{H}^{1 / 2}\left(\Gamma_{i}\right)$ and $k \in \mathbb{A}_{a d}$

$$
\begin{aligned}
& J_{1}(\eta, \tau, k)=\frac{\nu_{1}}{2}\left\|k \nabla\left(u_{1}\right) \cdot n-\phi\right\|_{\mathbb{H}^{-1 / 2}\left(\Gamma_{c}\right)}^{2}+\frac{1}{2}\left\|u_{1}-u_{2}\right\|_{\mathbb{H}^{1 / 2}\left(\Gamma_{i}\right)}^{2} \\
& J_{2}(\eta, \tau, k)=\frac{\nu_{2}}{2}\left\|u_{2}-f\right\|_{\mathbb{H}^{1 / 2}\left(\Gamma_{c}\right)}^{2}+\frac{1}{2}\left\|u_{1}-u_{2}\right\|_{\mathbb{H}^{1 / 2}\left(\Gamma_{i}\right)}^{2} \\
& J_{3}(\eta, \tau, k)=\left\|\sqrt{k} \nabla\left(\beta u_{1}+(1-\beta) u_{2}-u_{3}\right)\right\|_{\mathbb{L}^{2}(\Omega)}^{2}
\end{aligned}
$$

where $\nu_{1}$ and $\nu_{2}$ are the weights specific to each cost, and $\beta \in[0,1]$ is the weight in the cost $J_{3}$ dedicated to take into account a convex combination of both the solutions of (SP1) and (SP2). In practice, we have chosen $\nu_{1}=\nu_{2}=1$ and tried several values for $\beta$ in order to observe the impact on the computational efficiency of using both informations coming from $u_{1}$ and $u_{2}$.

For the data completion, the potential $\tau$ and the flux $\eta$ play a Nash game to minimize the Neumann gap $\left\|k \nabla\left(u_{1}\right) \cdot n-\phi\right\|_{\mathbb{H}^{-1 / 2}\left(\Gamma_{c}\right)}$ versus the Dirichlet gap $\left\|u_{2}-f\right\|_{\mathbb{H}^{1 / 2}\left(\Gamma_{c}\right)}$. The coupling term in common is the difference between $u_{1}$ and $u_{2}$ in $\Gamma_{i}$, which represents a regularization term. For the conductivity identification, the conductivity $k$ is used to minimize the Kohn-Vogelius cost $J_{3}$ to which we add a Tikhonov regularization term.

We shall say that there are three players. The first player controls the strategy variable $\eta$ which belongs to the space strategy $\mathbb{H}^{-1 / 2}\left(\Gamma_{i}\right)$, the second player controls the strategy variable $\tau$ which belongs to the space strategy $\mathbb{H}^{1 / 2}\left(\Gamma_{i}\right)$ and the third player controls the conductivity $k$ which belongs to $\mathbb{A}_{a d}$.

As a solution to our inverse problem, we seek a Nash equilibrium, defined as follows.

Definition 3.1. A triplet $\left(\eta_{N}, \tau_{N}, k_{N}\right) \in \mathbb{H}^{-1 / 2}\left(\Gamma_{i}\right) \times \mathbb{H}^{1 / 2}\left(\Gamma_{i}\right) \times \mathbb{A}_{a d}$ is a Nash equilibrium for the three players game involving the costs $J_{1}, J_{2}$ and $J_{3}$ if:

$$
\begin{cases}J_{1}\left(\eta_{N}, \tau_{N}, k_{N}\right) \leq J_{1}\left(\eta, \tau_{N}, k_{N}\right), & \forall \eta \in \mathbb{H}^{-1 / 2}\left(\Gamma_{i}\right), \\ J_{2}\left(\eta_{N}, \tau_{N}, k_{N}\right) \leq J_{2}\left(\eta_{N}, \tau, k_{N}\right), & \forall \tau \in \mathbb{H}^{1 / 2}\left(\Gamma_{i}\right), \\ J_{3}\left(\eta_{N}, \tau_{N}, k_{N}\right) \leq J_{3}\left(\eta_{N}, \tau_{N}, k\right), & \forall k \in \mathbb{A}_{a d} .\end{cases}
$$

In $[18,19]$, in the setting where the conductivity $k$ is fixed and known, the authors address the existence issue for the completion problem, and prove it by application of the Nash theorem. They established that the partial mapping $\eta \mapsto J_{1}(\eta, \tau, k)$ (resp. $\left.J_{2}\right)$ is a quadratic strongly convex functional over $\mathbb{H}^{-\frac{1}{2}}\left(\Gamma_{i}\right)$ (resp. $\mathbb{H}^{\frac{1}{2}}\left(\Gamma_{i}\right)$ ). This partial ellipticity property of $J_{1}$ holds uniformly w.r.t. $\tau$, and conversely for $J_{2}$. It allows to restrict the search for Nash equilibrium in data completion step (see step 1 of Algorithm 1) to bounded subsets of the strategy spaces, which remains consistent with the classical results of conditional stability of Cauchy problem (see e.g. [4]).

The existence of a Nash triplet is in turn much less straightforward to establish and is out of the scope of the present paper. The identification problem (finding the conductivity $k$ ) alone is quite harsh when only a single Cauchy data $(f, \Phi)$ is available $[17,23]$. 


\section{NumERICAL PROCEDURE}

We now present an algorithm dedicated to the computation of the Nash equilibrium solution to the coupled problem of data completion and conductivity identification. The gradient method with a fixed step is used to solve the problems of partial optimization of $J_{1}$ and $J_{2}$ and a Newton algorithm is used to minimize the functional $J_{3}$. The Nash-game algorithm is provided in Algorithm 1. We suppose that the measurements are noisy and we denote by $\sigma$ the level of noise. The relaxation parameter $t$ in the optimization loop is used to accelerate the convergence (see [19] and references therein). There are two loops in the algorithm, a main loop and a secondary one. The secondary loop denoted by (step 1) in the Algorithm 1 is for the optimization of the data completion problem given fixed conductivity values. It preconditions the update of the overall Nash iterates, by alternate minimization of functionals $J_{1}$ and $J_{2}$. The step 2 in the algorithm is for the optimization of the conductivities by minimizing the functional $J_{3}$ given the optimal data completion solution obtained from step 1. The overall stopping criteria for the algorithm is $\left\|u_{2}-f\right\|_{\mathbb{L}^{2}\left(\Gamma_{c}\right)} \leq \rho$, where $\rho$ depends on the measurement error (noise level) $\sigma$ (shortly speaking, we used a profile $\rho=\rho(\sigma)=a \sigma+b$ where the coefficients $a$ and $b$ are experimentally fitted, see $[2,19])$.

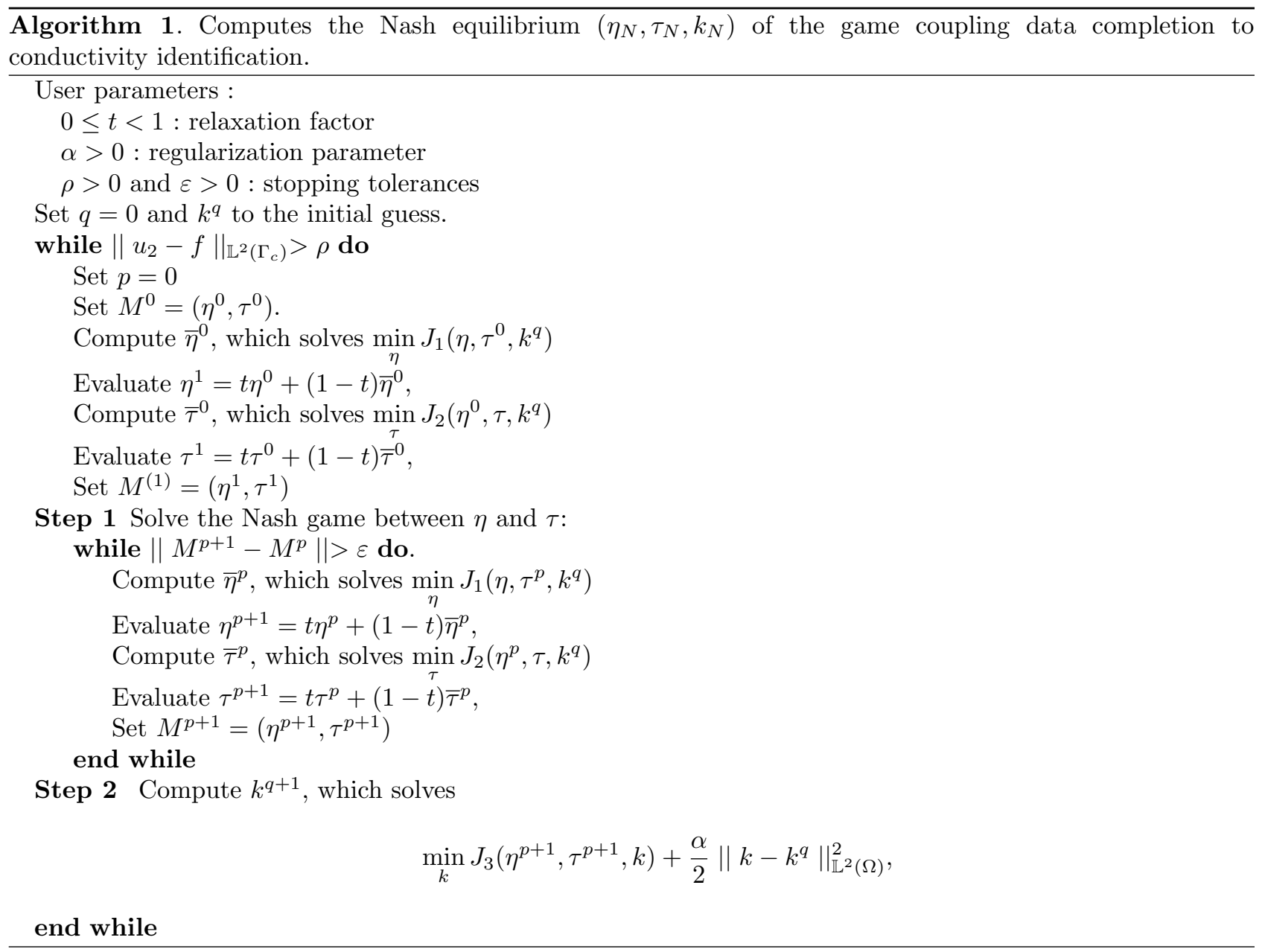

Let us remark that, in the Algorithm 1, along step 1, the players $J_{1}$ and $J_{2}$ play few iterations of a preconditioning Nash subgame, then they send their optimal strategies to the third player $J_{3}$. This algorithm actually computes a Nash equilibrium. A classical Nash algorithm would be that each of the three players minimizes 
in parallel its own cost, without the preconditioning step. Indeed, while it may look as a kind of Stackelberg game, it is not, since the third player does not use optimal response maps $\eta^{\text {opt }}(k), \tau^{\text {opt }}(k)$ from players $J_{1}$ and $J_{2}$ (which would be too expensive to determine). More precisely, player $J_{3}$ does not solve the program: $\min _{k} J_{3}\left(\eta^{\mathrm{opt}}(k), \tau^{\mathrm{opt}}(k), k\right)$.

In order to solve the minimization problems, we need to calculate the gradient of the costs $\nabla_{\eta} J_{1}, \nabla_{\tau} J_{2}$ and $\nabla_{k} J_{3}$, as well as the second derivative of $J_{3}$. For that, we used the Lagrangian method. To simplify the calculus of the derivatives of $J_{3}$, we choose in the following the coefficient $\beta$ equal to 0 .

Proposition 4.1. We have the following partial derivatives of $J_{1}$ and $J_{2}$ with respect to $\eta$ and $\tau$ :

$$
\begin{aligned}
& \left\{\begin{array}{l}
\frac{\partial J_{1}}{\partial \eta} \cdot \xi=-\int_{\Gamma_{i}} \lambda_{1} \xi \mathrm{d} \Gamma_{i}, \quad \forall \xi \in \mathbb{H}^{-1 / 2}\left(\Gamma_{i}\right) \\
\text { with } \lambda_{1} \in W_{1}=\left\{v \in \mathbb{H}^{1}(\Omega) \text { such that } v_{\mid \Gamma_{c}}=0\right\}, \text { solution of the adjoint problem: } \\
\int_{\Omega} k \nabla \lambda_{1} \nabla \gamma=-\int_{\Gamma_{c}}\left(k \nabla u_{1} . n-\phi\right) k \nabla \gamma \cdot n \mathrm{~d} \Gamma_{c}-\int_{\Gamma_{i}}\left(u_{1}-\tau\right) \gamma \mathrm{d} \Gamma_{i}, \quad \forall \gamma \in W_{1}
\end{array}\right. \\
& \left\{\begin{array}{l}
\frac{\partial J_{2}}{\partial \tau} \cdot h=\int_{\Gamma_{i}}\left(k \nabla \lambda_{2} . n-\left(u_{1}-\tau\right)\right) h \mathrm{~d} \Gamma_{i}, \quad \forall h \in \mathbb{H}^{1 / 2}\left(\Gamma_{i}\right) \\
\text { such that } \lambda_{2} \in \mathbb{H}^{1}(\Omega) \text { solution of the adjoint problem: } \\
\begin{cases}\operatorname{div}\left(k \nabla \lambda_{2}\right)=0 & \text { in } \Omega \\
\lambda_{2}=0 & \text { on } \Gamma_{i} \\
k \nabla \lambda_{2} . n=f-u_{2} & \text { on } \Gamma_{c}\end{cases}
\end{array}\right.
\end{aligned}
$$

For the resolution of the minimization problem of $J_{3}$ (step 2), we used the Newton algorithm. For that, we need to evaluate the first and the second derivatives of $J_{3}$ with respect to the conductivity.

Proposition 4.2. We suppose that $\Omega=\bigcup_{j=1}^{n} \Omega_{j}$ and the conductivity $k=k_{j}$ in $\Omega_{j}$. We define the spaces $\mathbb{H}_{0, \Gamma_{i}}^{1}(\Omega)=\left\{v \in \mathbb{H}^{1}(\Omega)\right.$ such that $\left.v_{\mid \Gamma_{i}}=0\right\}$ and $\mathbb{H}_{0}^{1}(\Omega)=\left\{v \in \mathbb{H}^{1}(\Omega)\right.$ such that $\left.v_{\mid \partial \Omega}=0\right\}$. The derivatives are the following:

(i) $\frac{\partial J_{3}}{\partial k_{j}} \cdot \varphi=\int_{\Omega_{j}}\left|\nabla\left(u_{2}-u_{3}\right)\right|^{2} \varphi \mathrm{d} \Omega+\int_{\Omega_{j}} \nabla u_{2} \nabla v_{2} \varphi \mathrm{d} \Omega+\int_{\Omega_{j}} \nabla u_{3} \nabla v_{3} \varphi \mathrm{d} \Omega, \quad \forall \varphi \in \mathbb{L}(\Omega)$ such that $v_{2} \in \mathbb{H}_{0, \Gamma_{i}}^{1}(\Omega)$ and $v_{3} \in \mathbb{H}_{0}^{1}(\Omega)$ are solutions of the following weak formulations adjoint problems:

$$
\begin{cases}\int_{\Omega} k \nabla v_{2} \nabla \gamma_{2}=-\int_{\Omega} 2 k \nabla\left(u_{2}-u_{3}\right) \nabla \gamma_{2} \mathrm{~d} \Omega, & \forall \gamma_{2} \in \mathbb{H}_{0, \Gamma_{i}}^{1}(\Omega), \\ \text { and } & \\ \int_{\Omega} k \nabla v_{3} \nabla \gamma_{3}=\int_{\Omega} 2 k \nabla\left(u_{2}-u_{3}\right) \nabla \gamma_{3} \mathrm{~d} \Omega, & \forall \gamma_{3} \in \mathbb{H}_{0}^{1}(\Omega) .\end{cases}
$$

(ii) $\frac{\partial^{2} J_{3}}{\partial k_{i} \partial k_{j}} \cdot\left(\varphi_{1}, \varphi_{2}\right)=\int_{\Omega_{i}} \nabla \gamma_{2} \nabla v_{2} \mathrm{~d} \Omega+\int_{\Omega_{i}} 2 \nabla\left(u_{2}-u_{3}\right) \nabla \gamma_{2} \varphi_{2} \mathrm{~d} \Omega-\int_{\Omega_{i}} 2 \nabla\left(u_{2}-u_{3}\right) \nabla \gamma_{3} \varphi_{2} \mathrm{~d} \Omega$ $+\int_{\Omega_{i}} \nabla u_{2} \nabla \xi_{2} \varphi_{2} \mathrm{~d} \Omega+\int_{\Omega_{i}} \nabla u_{3} \nabla \xi_{3} \varphi_{2} \mathrm{~d} \Omega, \quad \forall\left(\varphi_{1}, \varphi_{2}\right) \in \mathbb{L}(\Omega) \times \mathbb{L}(\Omega)$ such that $\left(\xi_{2}, \xi_{3}, \gamma_{2}, \gamma_{3}\right) \in \mathbb{H}_{0, \Gamma_{i}}^{1}(\Omega) \times \mathbb{H}_{0}^{1}(\Omega) \times \mathbb{H}_{0, \Gamma_{i}}^{1}(\Omega) \times \mathbb{H}_{0}^{1}(\Omega)$ are solutions of the following weak 
formulations adjoint problems:

$$
\left\{\begin{array}{l}
\int_{\Omega} k \nabla \xi_{2} \nabla w_{2}=-\int_{\Omega_{j}} 2 \nabla\left(u_{2}-u_{3}\right) \nabla w_{2} \varphi_{1} \mathrm{~d} \Omega-\int_{\Omega_{j}} \nabla w_{2} \nabla v_{2} \varphi_{1} \mathrm{~d} \Omega-\int_{\Omega} 2 k \nabla w_{2} \nabla \gamma_{2} \mathrm{~d} \Omega \\
+\int_{\Omega} 2 k \nabla w_{2} \nabla \gamma_{3}, \quad \forall w_{2} \in \mathbb{H}_{0, \Gamma_{i}}^{1}(\Omega), \\
\int_{\Omega} k \nabla \xi_{3} \nabla w_{3}=\int_{\Omega_{j}} 2 \nabla\left(u_{2}-u_{3}\right) \nabla w_{3} \varphi_{1} \mathrm{~d} \Omega-\int_{\Omega_{j}} \nabla w_{3} \nabla v_{3} \varphi_{1} \mathrm{~d} \Omega-\int_{\Omega} 2 k \nabla w_{3} \nabla \gamma_{3} \mathrm{~d} \Omega \\
+\int_{\Omega} 2 k \nabla w_{3} \nabla \gamma_{2}, \quad \forall w_{3} \in \mathbb{H}_{0}^{1}(\Omega), \\
\int_{\Omega} k \nabla \gamma_{2} \nabla h_{2}=-\int_{\Omega_{j}} \nabla u_{2} \nabla h_{2} \varphi_{1} \mathrm{~d} \Omega, \quad \forall h_{2} \in \mathbb{H}_{0, \Gamma_{i}}^{1}(\Omega), \\
\text { and } \\
\int_{\Omega} k \nabla \gamma_{3} \nabla h_{3}=-\int_{\Omega_{j}} \nabla u_{3} \nabla h_{3} \varphi_{1} \mathrm{~d} \Omega, \quad \forall h_{3} \in \mathbb{H}_{0}^{1}(\Omega) .
\end{array}\right.
$$

Remark 4.3. From our numerical experiments, we noticed that the standard Nash algorithm takes a long time to converge to the equilibrium. To save time and speed up the convergence, we limit the number of iterations in the data completion part (step 1), then we proceed to the conductivity identification procedure (step 2).

\section{NUMERICAL RESUlTS: ANATOMICAL MODEL}

Our main code is implemented using FreeFem $++[21]$, a powerful language and environment that allows the numerical solution of partial differential equations using the finite element method.

Since we target electrocardiography applications, we will present two situations, in a 2D realistic model of the thorax. The boundary of the domain is divided into two parts. The outer boundary of the thorax is the accessible part $\Gamma_{c}$ where the Cauchy data are available and the heart boundary is the inner inaccessible $\Gamma_{i}$ where the electrical potential and the flux are missing. While the functional spaces $\mathbb{H}^{-1 / 2}\left(\Gamma_{i}\right) \times \mathbb{H}^{1 / 2}\left(\Gamma_{i}\right)$ dedicated to the over specified data $(f, \Phi)$ are well adapted to our theoretical framework, we have led computational experiments with much more regular given data $(e . g . \Phi=0)$. However, to assess the stability of the algorithm, we have considered perturbations of the electric potential $f$ that belong only to $L^{2}\left(\Gamma_{c}\right)$.

Each part of the torso is characterized by a conductivity coefficient, such that we know only an approximate value of it. Our goal is to find the electrical potential and the flux on the surface and enhance the conductivity estimation in the domain.

\subsection{Problem setting: computational domain and exact solution}

To perform the computations, we need to distinguish the different regions in the computational domain for which we aim to estimate the conductivity values. To do that, we segmented a two-dimension slice of a magnetic resonance image (MRI). The MRI measures the diffusion of water molecules in biological tissues, which is useful to distinguish different regions in the torso domain. We segmented the image in Figure 2 (left) inorder to construct the different regions. In our study, we will consider four organs: the heart surface, lungs, muscles (cavity) and the fat. After the segmentation, we construct a 2D mesh of the torso cross section in which we identify the organs as shown in Figure 2 (right). In this representation, we consider that all the cavity region is occupied by the muscles. The 2D mesh contains 2395 vertices and 4540 elements. Each layer is caracterized by a constant conductivity:

$$
k^{e x}= \begin{cases}k_{1}=1, & \text { in } \Omega_{1}=\text { fat } \\ k_{2}=1, & \text { in } \Omega_{2}=\text { cavity } \\ k_{3}=0.8, & \text { in } \Omega_{3}=\text { lung }\end{cases}
$$



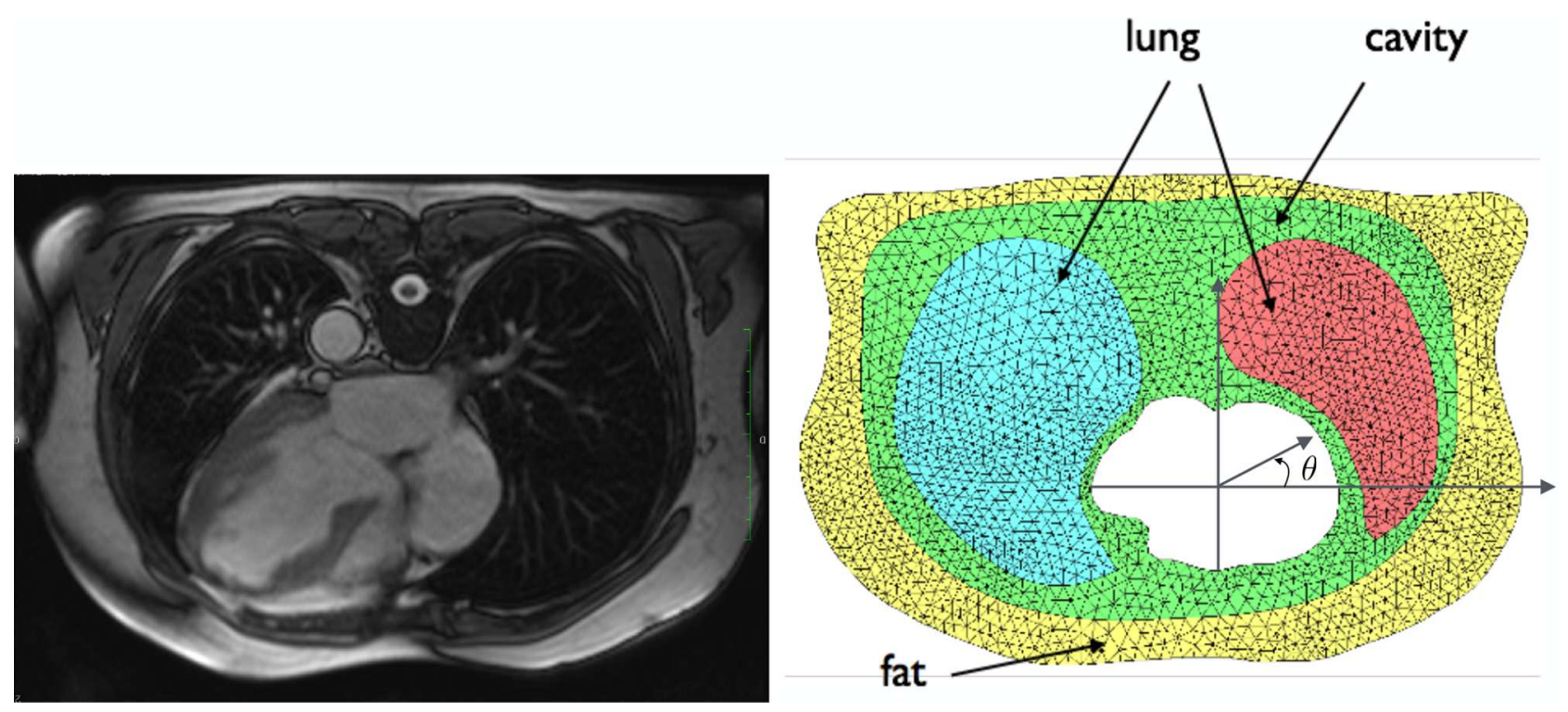

Figure 2. MRI 2D slice of the torso (left), 2D computational mesh of the torso geometry showing the different regions of the torso considered in this study: fat, lungs and torso cavity $($ right). The angle $\theta$ is the second polar coordinate.

The outer boundary of the thorax plays the role of $\Gamma_{c}$, where the Cauchy data are over specified. The heart's boundary plays the role of $\Gamma_{i}$, where the potential and the flux are missing. We parametrize the one-dimensional heart boundary with the $\theta$ variable, in order to be able to represent both the incomplete Dirichlet and Neumann boundary conditions on the heart interface (see Fig. 2 (right)). The Cauchy data $f$ and $\phi$ are generated via the solution of the following direct problem:

$$
\begin{cases}\operatorname{div}\left(k^{e x} \nabla(u)\right)=0, & \text { in } \Omega, \\ u=e^{x} \cos (y), & \text { on } \Gamma_{i}, \\ k^{e x} \nabla(u) \cdot n=\phi=0, & \text { on } \Gamma_{c} .\end{cases}
$$

We use standard value of the different conductivities as initial guess but also we use them in the regularization. During iterations, we choose a value of the parameters $\alpha$ and $t$, such that $\alpha$ worth $1.5 \times 10^{-4}$ in test case 1 for noise-free and $8 \times 10^{-4}$ for noisy Cauchy data. In test case $2, \alpha$ worth $5 \times 10^{-4}$. The parameter $t$ is equal to 0.1 in both tests.

\subsection{Test case 1}

In this test case, we would like to show how optimizing the conductivities improves the solution of the inverse problem. The idea is to, first, solve the data completion problem where the conductivities are not exact which is the case in the studies done in the literature. We will refer to these conductivities by measured conductivity. Second, we will solve the coupled problem by optimizing the conductivity while solving the data completion problem. This will show the gain that we obtain by solving the coupled problem.

The measured conductivity is taken to be $k_{3}^{0}=0.5$ while the exact conductivity $k_{3}=0.8$. We solve the data completion problem which consists of reconstructing the potential and the flux on $\Gamma_{i}$ using the Nash algorithm 

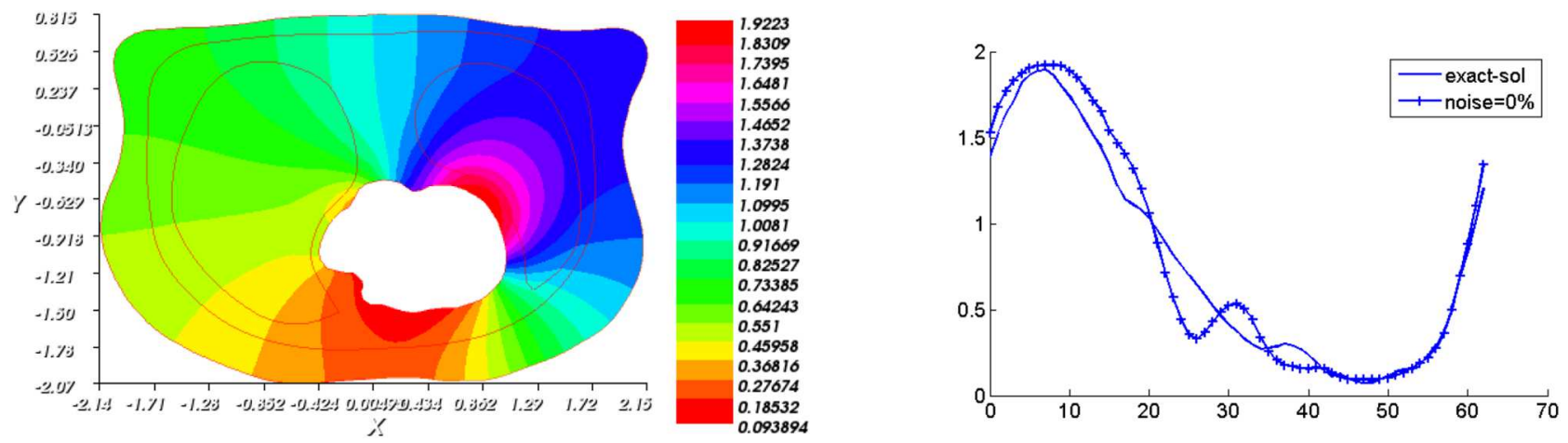

Figure 3. Reconstructed potential $u_{1}$ (left) and reconstructed Dirichlet condition (right) compared to the exact solution on $\Gamma_{i}$ using an approximate value of $k_{3}=k_{3}^{0}=0.5$.

(step 1 of our algorithm). During iterations, the conductivity is kept equal to the measured conductivity:

$$
k^{0}= \begin{cases}k_{1}=1, & \text { in } \Omega_{1}=\text { fat } \\ k_{2}=1, & \text { in } \Omega_{2}=\text { cavity } \\ k_{3}^{0}=0.5, & \text { in } \Omega_{3}=\text { lung }\end{cases}
$$

We start from an initial guess $\left(\eta^{0}, \tau^{0}\right)=(0,0)$. Figure 3 presents the reconstructed potential over $\Gamma_{i}$ compared to the exact solution and the optimal solution $u_{1}^{\mathrm{opt}}:=u_{1}\left(\eta^{\mathrm{opt}}, k^{0}\right)$. At convergence, the relative error $\frac{\left\|\tau^{\mathrm{opt}}-u^{\mathrm{ex}}\right\|_{\mathbb{L}^{2}\left(\Gamma_{i}\right)}}{\left\|u^{\mathrm{ex}}\right\|_{\mathbb{L}^{2}\left(\Gamma_{i}\right)}}$ is equal to 0.13 and $\frac{\left\|u_{1}^{\mathrm{opt}}-u^{\mathrm{ex}}\right\|_{\mathbb{L}^{2}(\Omega)}}{\left\|u^{\mathrm{ex}}\right\|_{\mathbb{L}^{2}(\Omega)}}$ is 0.04 , where $u^{\mathrm{ex}}$ is the solution of (5.1).

To obtain a better reconstruction, we applied our algorithm, presented in Section 4, to enhance the value of the conductivity and therefore to improve the data completion. The value of the initial estimation is $\left(\eta^{0}, \tau^{0}, k_{3}^{0}\right)=(0,0,0.5)$. Figure 5 presents the reconstructed potential on $\Gamma_{i}$, compared to the exact solution. We also show the optimal solution $u_{1}$ in the thorax and the evolution of the reconstructed conductivity during the Nash iterations. At convergence, the obtained conductivity $k_{3}^{\mathrm{opt}}$ is equal to 0.794542 and the relative errors $\frac{\left\|\tau^{\mathrm{opt}}-u^{\mathrm{ex}}\right\|_{\mathbb{L}^{2}\left(\Gamma_{i}\right)}}{\left\|u^{\mathrm{ex}}\right\|_{\mathbb{L}^{2}\left(\Gamma_{i}\right)}}, \frac{\left\|u_{1}^{\mathrm{opt}}-u^{\mathrm{ex}}\right\|_{\mathbb{L}^{2}(\Omega)}}{\left\|u^{\mathrm{ex}}\right\|_{\mathbb{L}^{2}(\Omega)}}$ are equal to 0.032 and 0.0054 , respectively.

We repeated the same test but using two different meshes. The first Mesh is coarser it contains 2206 vertices and the second is finer and it contains 17432 vertices. Figures of both meshes are depicted in Figure 4. At convergence, for the coarse (respectively, fine) mesh, the obtained conductivity worth 0.84 (respectively, 0.752 ) and the relative error on the Dirichlet part of the solution is equal to 0.026 (respectively, 0.035). In the three different degrees of refinement of the computational geometry the relative error on the conductivity does not exceed $6 \%$ and the relative error on the reconstructed electrical potentiel (Dirichlet condition) does not exceed $5 \%$.

We show in Figure 3 the results of a recovered Dirichlet data obtained for an approximate or initial knowledge of the conductivity parameter. Then, in Figure 5, we present the results of the recovered Dirichlet data for an optimally identified conductivity. It can be easily observed from these two results how critical is a good identification of the conductivities on the quality of the recovery of the data missing on the inaccessible boundary.

In Figure 6, we present the behavior of the three functionals during the first 100 iterations. From the curves, it seems that the values are very close to zero near the 100th iteration, but to rach the optimal solution, we continue for more iterations. To assess the robustness of our numerical procedure, we disturb the Cauchy data by adding some noise. Figure 7 shows the reconstructed potential on $\Gamma_{i}$ compared to the exact one and the behavior of the conductivity $k_{3}$ during the optimization process for different noise levels. The estimated value of 

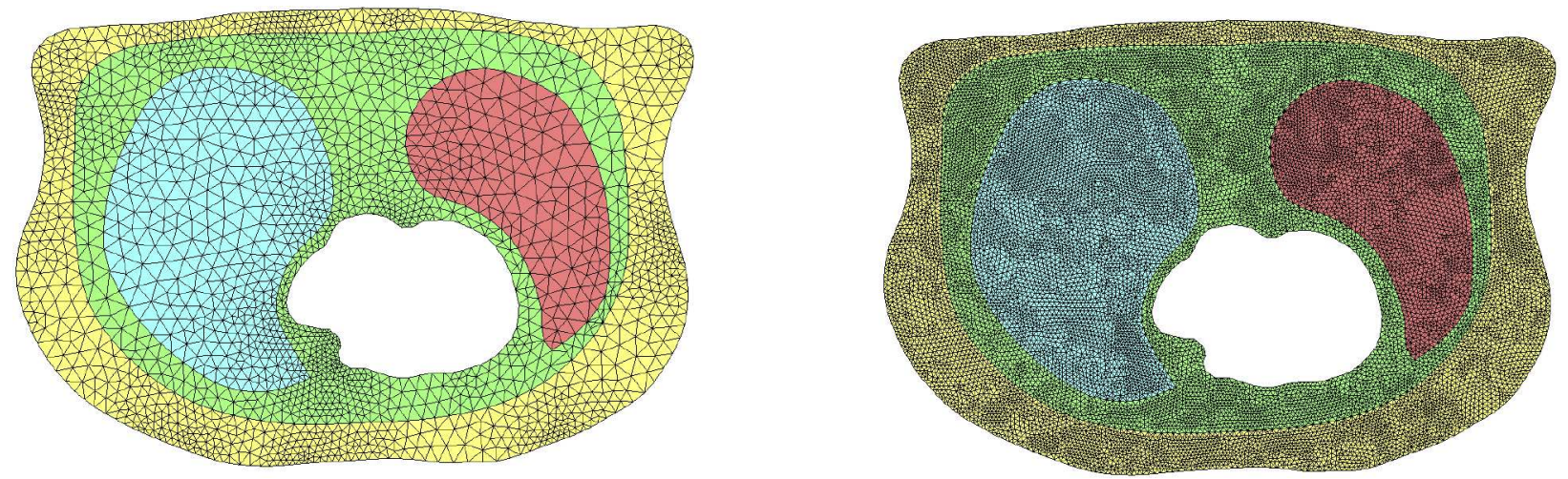

Figure 4. Two different degrees of refinement of the torso mesh. The corser mesh (left) has 2206 vertices and the finer mesh (right) has 17432 vertices.
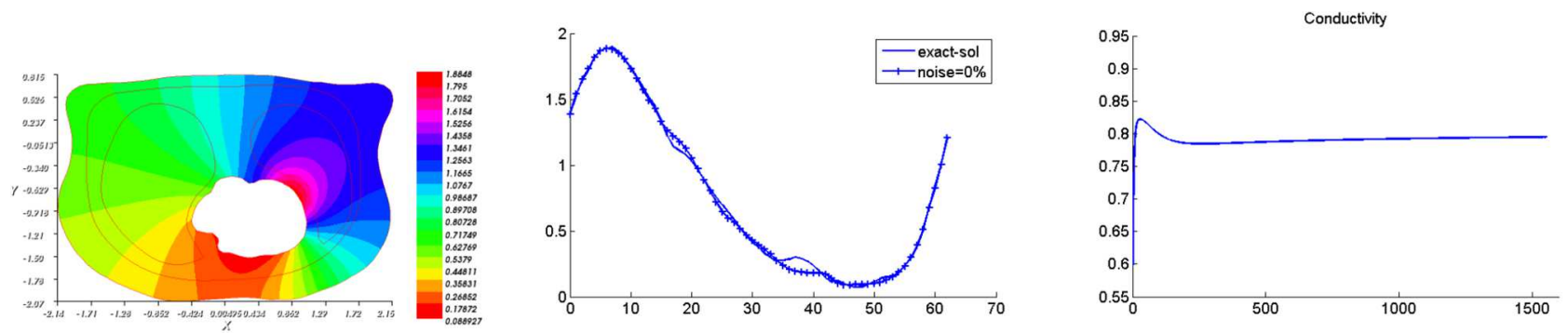

Figure 5. Reconstructed potential $u_{1}$ (left) and reconstructed dirichlet condition (middle) compared to the exact solution on $\Gamma_{i}$ for noise-free. The behavior of the conductivity during iterations is also plotted (right). At convergence, the reconstructed conductivity $k_{3}^{\text {opt }}=$ 0.794542 .
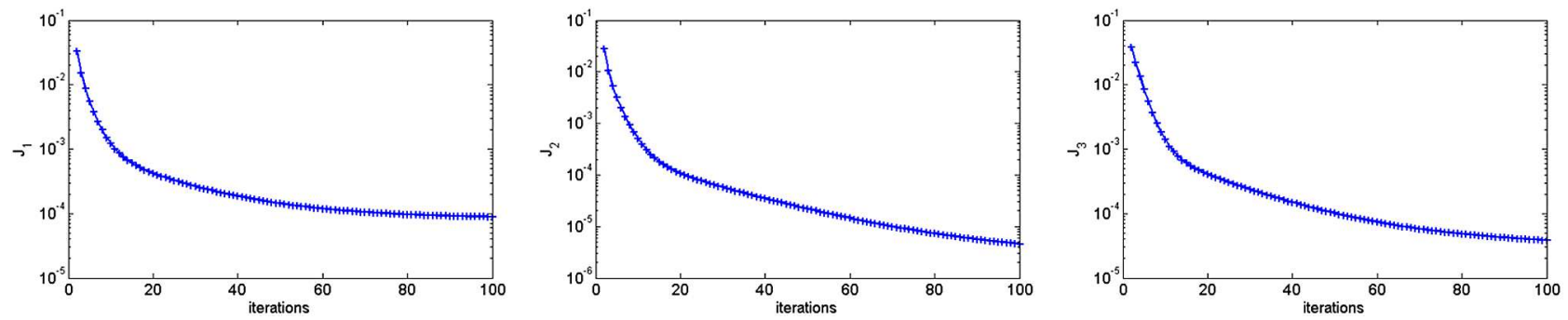

Figure 6 . The behavior of the costs $J_{1}$ (left), $J_{2}$ (middle) and $J_{3}$ (right) during the first 100 iterations.

$k_{3}^{\text {opt }}$ at convergence is $0.7752,0.8236$ and 0.8733 for $1 \%, 3 \%$ and $5 \%$ noise levels, respectively. In order to show the performance of our method, we compute different error indicators. The numerical results are presented in Table 1. We remark that the relative error on the estimated conductivity is much more sensitive to the noise level than it is for the reconstructed solution.

For the sake of proving the choice of $\alpha$, we present in Figure 8 the error of conductivity $\frac{\left\|k^{\mathrm{ex}}-k^{\mathrm{opt}}\right\|_{2}}{\left\|k^{\mathrm{ex}}\right\|_{2}}$ and the error of dirichlet $\frac{\left\|\tau^{\mathrm{opt}}-u^{\mathrm{ex}}\right\|_{\mathscr{L}^{2}\left(\Gamma_{i}\right)}}{\left\|u^{\mathrm{ex}}\right\|_{\mathrm{L}^{2}\left(\Gamma_{i}\right)}}$ with respect to different values of $\alpha$. We notice from the curves that the optimal parameter is between $10^{-4}$ and $3 \times 10^{-3}$ for noise free (Identically in the case of $3 \%$ of noise). 

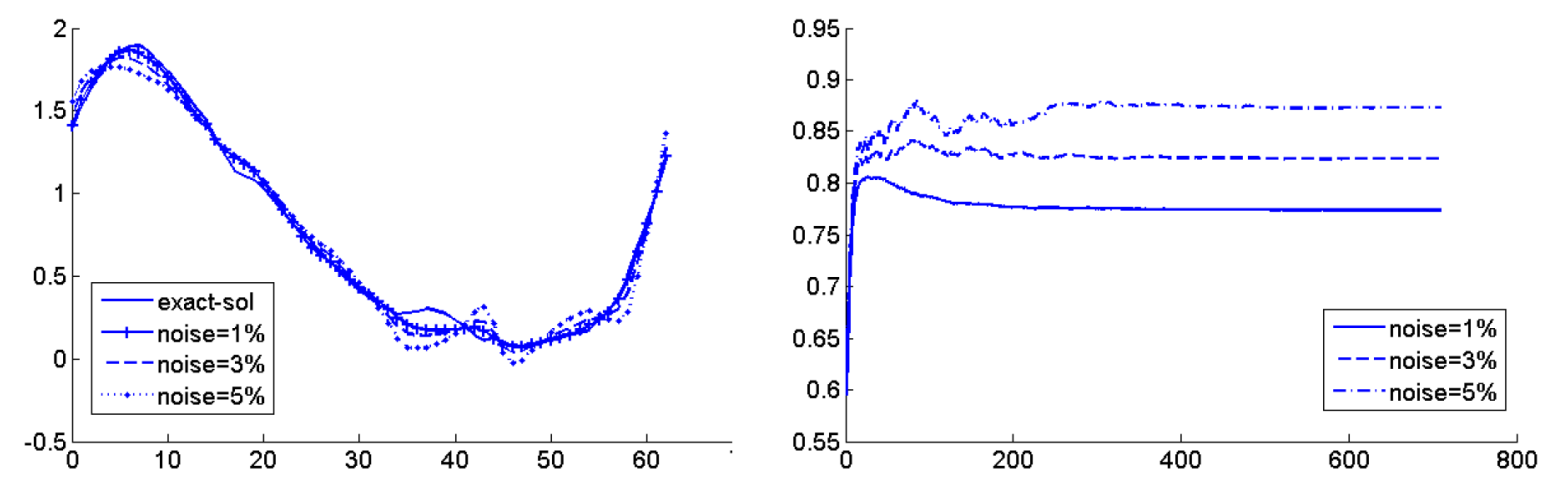

Figure 7. The reconstructed dirichlet condition compared to the exact solution on $\Gamma_{i}$ for different noise levels $(1 \%, 3 \%, 5 \%)$ (left). The behavior of the conductivity during iterations of the optimization algorithm $($ right $)$.

TABLE 1. The effect of Cauchy data noise level on the reconstructed solution: relative errors of reconstructed solution on the heart boundary and in the whole domain, and relative error of the estimated $k_{3}$ with respect of the noise level.

\begin{tabular}{|c|c|c|c|c|}
\hline \multirow{2}{*}{ Noise level } & $\left\|\tau^{\mathrm{opt}}-u^{\mathrm{ex}}\right\|_{\mathbb{L}^{2}\left(\Gamma_{i}\right)}$ & $\left\|u_{1}^{\mathrm{opt}}-u^{\mathrm{ex}}\right\|_{\mathbb{L}^{2}(\Omega)}$ & $\left\|u_{1}^{\mathrm{opt}}-u_{2}^{\mathrm{opt}}\right\|_{\mathbb{L}^{2}(\Omega)}$ & $\left\|k^{\mathrm{ex}}-k^{\mathrm{opt}}\right\|_{2}$ \\
\hline & $\left\|u^{\mathrm{ex}}\right\|_{\mathbb{L}^{2}\left(\Gamma_{i}\right)}$ & $\left\|u^{\mathrm{ex}}\right\|_{\mathbb{L}^{2}(\Omega)}$ & $\left\|u^{\mathrm{ex}}\right\|_{\mathbb{L}^{2}(\Omega)}$ & $\left\|k^{\mathrm{ex}}\right\|_{2}$ \\
\hline $0 \%$ & 0.032 & 0.0054 & 0.00015 & 0.006 \\
\hline $1 \%$ & 0.038 & 0.008 & 0.0011 & 0.0309 \\
\hline $3 \%$ & 0.066 & 0.017 & 0.0034 & 0.029 \\
\hline $5 \%$ & 0.11 & 0.028 & 0.0061 & 0.091 \\
\hline
\end{tabular}
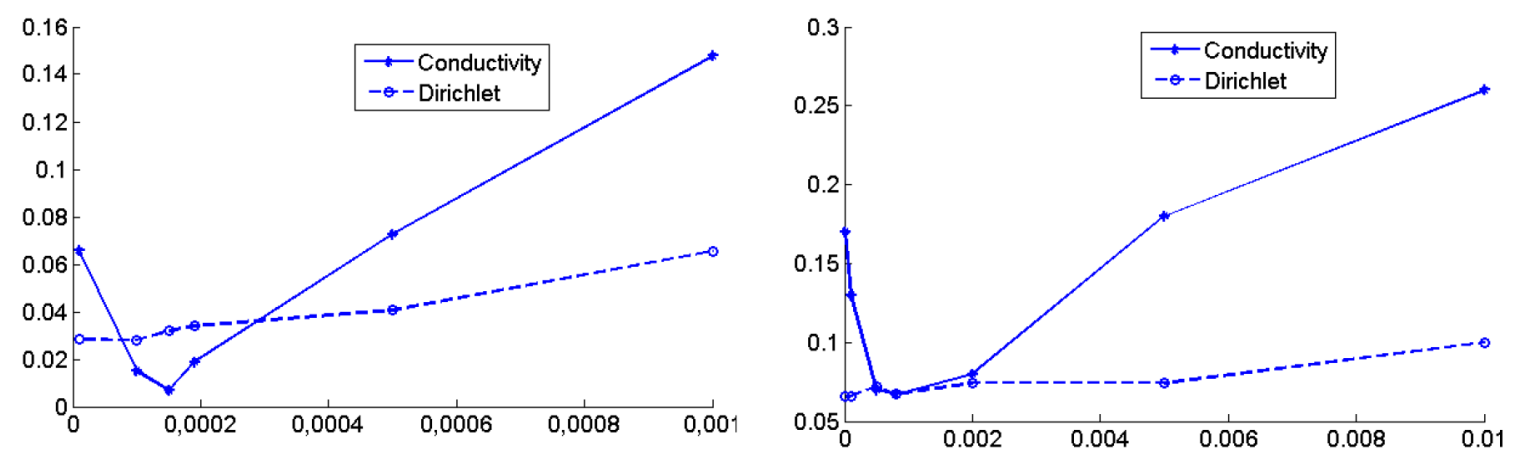

FIGURE 8. The conductivity error $\frac{\left\|k^{\mathrm{ex}}-k^{\mathrm{opt}}\right\|_{2}}{\left\|k^{\mathrm{ex}}\right\|_{2}}$ and Dirichlet error $\frac{\left\|\tau^{\mathrm{opt}}-u^{\mathrm{ex}}\right\|_{\mathbb{L}^{2}\left(\Gamma_{i}\right)}}{\left\|u^{\mathrm{ex}}\right\|_{\mathbb{L}^{2}\left(\Gamma_{i}\right)}}$ are presented with respect to different values of $\alpha$ for noise free (left) and for $3 \%$ of noise (right).

\subsection{Test case 2}

In this test case, we consider the same thorax, but we suppose that the patient has a pathological condition affecting the conductivities in the lung so that the right and left parts of the lung have different constant conductivity values. We split the thorax into four layers: fat, cavity, the right and the left part of the lung. The 
(a)
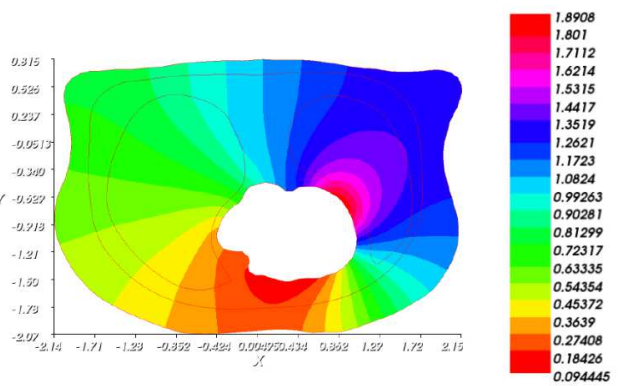

(c)

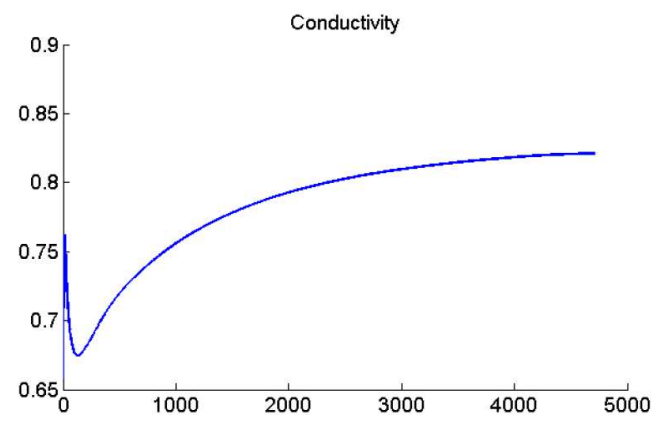

(b)

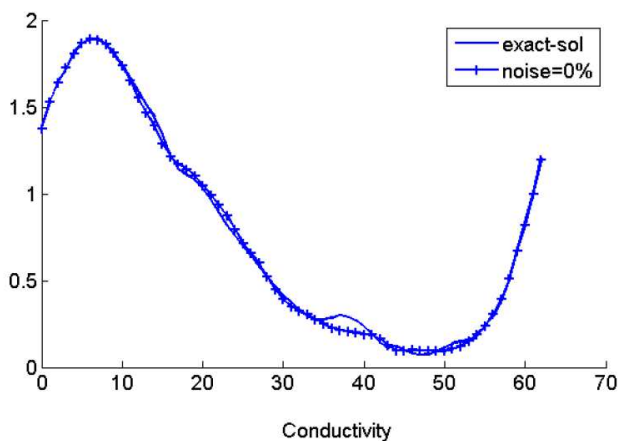

(d)

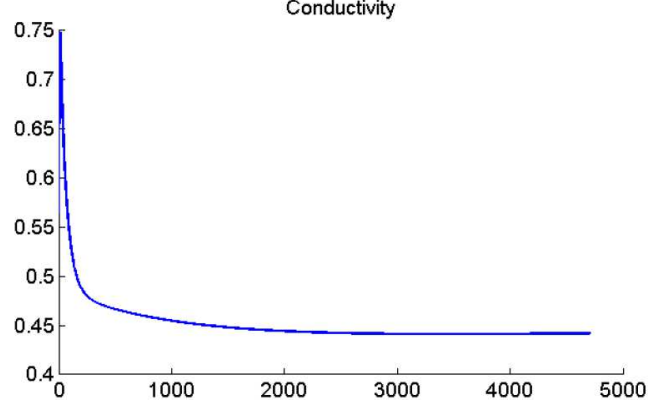

Figure 9. (a) Reconstructed potential $u_{1}$ in the domain, (b) reconstructed Dirichlet condition on $\Gamma_{i}$. (c) The behavior of the conductivities $k_{3}$ and (d) $k_{4}$ during iterations for noise free. At convergence, the conductivities are equal to $k_{3}^{\mathrm{opt}}=0.821142$ and $k_{4}^{\mathrm{opt}}=0.441652$.

conductivity in the domain follows the same decomposition, being constant in each of the four subdomains.

$$
k^{\mathrm{ex}}= \begin{cases}k_{1}=1 & \text { in } \Omega_{1}=\text { fat } \\ k_{2}=1 & \text { in } \Omega_{2}=\text { cavity } \\ k_{3}=0.8 & \text { in } \Omega_{3}=\text { right lung } \\ k_{4}=0.4 & \text { in } \Omega_{4}=\text { left lung }\end{cases}
$$

The Cauchy data are numerically simulated, as in the first test case, by solving the following direct problem:

$$
\begin{cases}\operatorname{div}\left(k^{\operatorname{ex}} \nabla(u)\right)=0 & \text { in } \Omega, \\ u=e^{x} \cos (y) & \text { on } \Gamma_{i}, \\ k^{\operatorname{ex}} \nabla(u) \cdot n=\phi=0 & \text { on } \Gamma_{c} .\end{cases}
$$

We suppose that we have an approximate value of the conductivities $k_{3}$ and $k_{4}$. Our goal is to enhance those measurements and find the potential and flux on the surface. We start from an initial guess $\left(\eta^{0}, \tau^{0}, k_{3}^{0}, k_{4}^{0}\right)=$ $(0,0,0.6,0.6)$. We show in Figure 9 the reconstructed dirichlet condition on $\Gamma_{i}$ compared to the exact one, the reconstructed potential $u_{1}$ in the domain and the behavior of the conductivities during Nash iterations for noise free data.

At convergence, the reconstructed conductivities are equal to $k_{3}^{\text {opt }}=0.821142$ and $k_{4}^{\text {opt }}=0.441652$. In Figure 10, we present the same reconstructions illustrating the stability of our method with respect to noisy data. The reconstructed $k_{3}^{\text {opt }}$ was $0.71969,0.831021$ and 0.912696 for $1 \%, 3 \%$ and $5 \%$ of noise levels respectively and $k_{4}^{\text {opt }}$ was $0.5079,0.510126$ and 0.506341 respectively for the same noise levels. Table 2 shows different errors for different noise levels. 

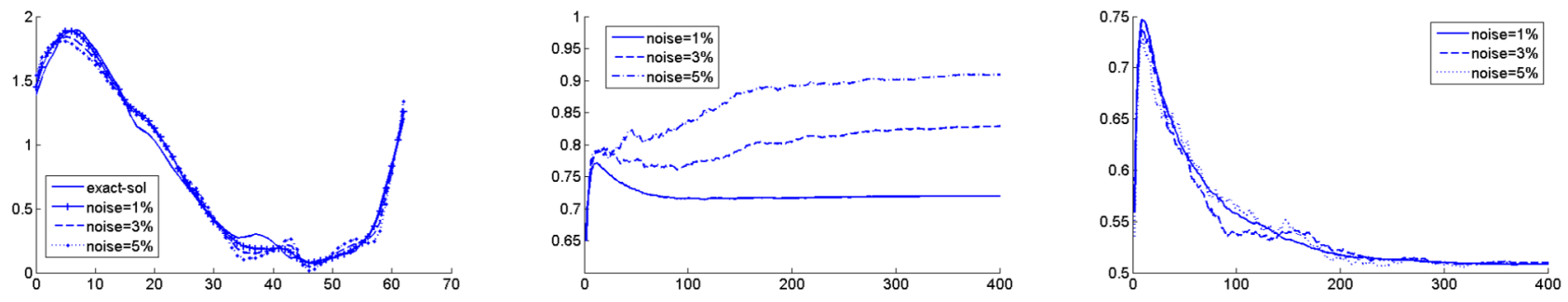

FIGURE 10. Reconstructed dirichlet condition on $\Gamma_{i}(l e f t)$ and the behavior of the conductivities $k_{3}$ (middle) and $k_{4}$ (right) during iterations for different noise levels $(1 \%, 3 \%, 5 \%)$.

TABLE 2. The effect of Cauchy data noise level on the reconstructed solution: relative errors of reconstructed solution on the heart boundary and in the whole domain, and relative error of the estimated couple $\left(k_{3}, k_{4}\right)$ with respect of the noise level.

\begin{tabular}{ccccc}
\hline \multirow{2}{*}{ Noise level } & $\left\|\tau^{\mathrm{opt}}-u^{\mathrm{ex}}\right\|_{\mathbb{L}^{2}\left(\Gamma_{i}\right)}$ & $\frac{\left\|u_{1}^{\mathrm{opt}}-u^{\mathrm{ex}}\right\|_{\mathbb{L}^{2}(\Omega)}}{\left\|u^{\mathrm{ex}}\right\|_{\mathbb{L}^{2}\left(\Gamma_{i}\right)}}$ & $\frac{\left\|u_{1}^{\mathrm{opt}}-u_{2}^{\mathrm{opt}}\right\|_{\mathbb{L}^{2}(\Omega)}}{\left\|u^{\mathrm{ex}}\right\|_{\mathbb{L}^{2}(\Omega)}}$ & $\frac{\left\|k^{\mathrm{ex}}-k^{\mathrm{opt}}\right\|_{2}}{\left\|u^{\mathrm{ex}}\right\|_{\mathbb{L}^{2}(\Omega)}}$ \\
\hline $0 \%$ & 0.027 & 0.0054 & 0.00017 & 0.05 \\
$1 \%$ & 0.051 & 0.012 & 0.0011 & 0.15 \\
$3 \%$ & 0.07 & 0.018 & 0.0035 & 0.12 \\
$5 \%$ & 0.09 & 0.026 & 0.0059 & 0.17 \\
\hline
\end{tabular}

\section{Conclusion}

The electrocardiography imaging technique is widely used to detect cardiac pathologies. The mathematical problem behind this technology is to solve the data completion problem of the diffusion equation where the measurement of the electrical potential on the torso surface and the null flux boundary conditions are considered as the Cauchy data. Beside the ill-posedness of the mathematical problem this technology is still not fully accurate, mainly because of the fact that many aspects of the electrophysiology of the underlying problem are not accurately described. In particular, the heterogeneity of the torso conductivities is usually neglected. In this paper, we presented an approach to solve the coupled problem by solving the data completion and optimizing the conductivities of the organs in the torso using an original Nash game strategy. Three players are defined which play non-cooperatively a Nash game: the two first players dedicate their costs to the completion problem, while the third one cost is related to the conductivity identification problem. We setup the game framework, and present the algorithm which is implemented using Freefem ++ . We conducted two numerical experiments, assessing the case where the measured data are disturbed by random noise as well. The obtained results prove that our algorithm is efficient in performing the coupled completion/identification tasks, and is stable with respect to noisy data.

Acknowledgements. This work has been supported by EPICARD cooperative research program, funded by INRIA international laboratory LIRIMA. The LAMSIN researcher's work is supported on a regular basis by the Tunisian Ministry of Higher Education, Scientific Research and Technology. This study also received financial support from the French Government as part of the 'Investments of the future' program managed by the National Research Agency (ANR), Grant reference ANR-10-IAHU-04.

\section{REFERENCES}

[1] R. Aboulaich, A. Ben Abda and M. Kallel, Missing boundary data reconstruction via an approximate optimal control. Inverse Probl. Imaging 2 (2008) 411-426.

[2] R. Aboulaich, A. Ben Abda and M. Kallel, A control type method for solving the Cauchy-Stokes problem. Appl. Math. Model. 37 (2013) 4295-4304. 
[3] R. Aboulaich, N. Fikal, E.M. El Guarmah and N. Zemzemi, Stochastic finite element method for torso conductivity uncertainties quantification in electrocardiography inverse problem. MMNP 11 (2016) 1-19.

[4] G. Alessandrini, L. Rondi, E. Rosset and S. Vessella, The stability for the Cauchy problem for elliptic equations. Inverse Probl. 25 (2009) 123004.

[5] S. Andrieux, A. Ben Abda and T.N. Baranger, Solving Cauchy problems by minimizing an energy-like functional. Inverse Probl. 22 (2006) 115-133.

[6] F.D. Araruna, E. Fernandez-Cara, S. Guerrero and M.C. Santos, New results on the Stacklberg, Nash exact control of linear parabolic equations. Syst. Control Lett. 104 (2017) 78-85.

[7] L. Bear, R. Dubois and N. Zemzemi, Optimization of organ conductivity for the forward problem of electrocardiography. Computing in Cardiology Conference (CinC) (2016) 385-388.

[8] F. Ben Belgacem and H. El Fekih, On Cauchy's problem: I. A variational Steklov-Poincaré theory. Inverse Probl. 21 (2005) $1915-1936$.

[9] F. Ben Belgacem, H. El Fekih and F. Jelassi, The Lavrentiev regularization of the data completion problem. Inverse Probl. 24 (2008) 9-45.

[10] M. Boulakia, M. Fernández, J. Gerbeau and N. Zemzemi, Direct and inverse problems in electrocardiography. AIP Conf. Proc. 1048 (2008) 113-117.

[11] R. Brown, Recovering the conductivity at the boundary from Dirichlet to Neumann map: a point wise result. J. Inverse Ill-posed Probl. 9 (2013) 567-574.

[12] A. Cimetière, F. Delvare, M. Jaoua and F. Pons, Solution of the Cauchy problem using iterative Tickhnov regularization. Inverse Probl. 17 (2001) 553-570.

[13] Daniel and Tataru, Unique continuation for solutions to pde's; between hörmander's theorem and holmgren' theorem. Commun. Partial Differ. Equ. 20 (1995) 855-884.

[14] K.R. Foster and H.P. Schwan, Dielectric properties of tissues and biological materials: a critical review. Crit. Rev. Biomed. Eng. 17 (1988) 25-104.

[15] S. Gabriel, R. Lau and C. Gabriel, The dielectric properties of biological tissues: ii. measurements in the frequency range 10 Hz to $20 \mathrm{GHz}$. Phys. Med. Biol. 41 (1996) 22-51.

[16] S.E. Geneser, R.M. Kirby and R.S. MacLeod, Application of stochastic finite element methods to study the sensitivity of ECG forward modeling to organ conductivity. IEEE Trans. Biomed. Eng. 55 (2008) 31-40.

[17] S. Gutman and J. Ha, Identifiability of piecewise constant conductivity in a heat conduction process. SIAM J. Control Optim. 64 (2007) 694-713.

[18] A. Habbal and M. Kallel, Data completion problems solved as Nash games. J. Phys.: Conf. Ser. 386 (2012) 012004.

[19] A. Habbal and M. Kallel, Neumann-Dirichlet Nash strategies for the solution of elliptic Cauchy problems. SIAM J. Control Optim. 51 (2013) 4066-4083.

[20] J. Hadamard, Lectures on Cauchy's Problem in Linear Partial Differential Equation. Dover, New York (1953).

[21] F. Hecht, A. Le Hyaric and O. Pionneau, Freefem++, Available at: http://www.freefem.org/ff++/

[22] M. Kallel, M. Moakher and A. Theljani, The Cauchy problem for a nonlinear elliptic equation: Nash-game approach and application to image inpainting. Inverse Probl. Imaging 9 (2015) 853-874.

[23] R. Kohn and M. Vogelius, Identification of an unknown conductivity by means of measurements at the boundary. SIAM AMS Proc. 14 (1984) 113-123.

[24] V.A. Kozlov, V.G. Maz'ya and A.V. Fomin, An iterative method for solving the Cauchy problem for elliptic equations. Comput. Math. Math. Phys. 31 (1991) 45-52.

[25] A. Nachman, A. Tamasan and A. Timonov, Recovering the conductivity from a single measurement of interior data. Inverse Probl. 25 (2009) 035014.

[26] L. Päivärinta, A. Panchenko and G. Uhlmann, Complex geometrical optics solutions for Lipschitz conductivities. Rev. Mat. Iberoam. 19 (2003) 57-72.

[27] J. Sylvester and G. Uhlmann, A global uniqueness theorem for an inverse boundary value problem. Ann. Math. 125 (1987) $153-169$.

[28] A. Van Oosterom and G.J. Huiskamp, The effect of torso inhomogeneities on body surface potentials quantified using a, tailored geometry. J. Electrocardiol. 22 (1989) 53-72.

[29] F.M. Weber, D.U. Keller, S. Bauer, G. Seemann, C. Lorenz and O. Dossel, Predicting tissue conductivity influences on body surface potentialsan efficient approach based on principal component analysis. IEEE Trans. Biomed. Eng. 58 (2011) 256-273. 